\title{
新しい応力関数を使用するせん断変形を含む平面板のフーリエ級数解 FOURIER SOLUTION OF NEW STRESS FUNCTIONS IN A THEORY OF PLATE BENDING INCLUDING THE EFFECT OF TRANSVERSE SHEAR DEFORMATION
}

\author{
末 岡禎佑 ${ }^{*}$, 古賀 掲維**, 近藤一夫*** \\ Teisuke SUEOKA, Aoi KOGA and Kazuo KONDOH.
}

\begin{abstract}
New stress functions introduced by prof. Tosaka and prof. Sueoka for expressing stress components at a point in three dimensional elastic body are used in a theory of plate bending including the effect of transverse shear deformation. Two conditions of compatibility are obtained as governing differential equations expressed by new stress functions.

Displacement components and resultant stress components acting at a point of section of the plate obtained by employing double trigonometric series agree with these obtained by using displacement method in expression.

It is shown that numerical results of displacements, bending moments and shearing forces for a uniformly loaded rectangular plate with two opposite edges simply supported, the third edge clamped, and the forth edge free, agree with calculated results by employing displacement method.
\end{abstract}

\author{
Keyword: plate, shear deformation, Hellinger-Reissner principle, conditions of compatibility, \\ stress functions, Fourier series \\ 板, せん断変形, Hellinger-Reissner の原理, 適合条件, 応力関数, フーリエ級数
}

\section{1..はじめに}

平面応力理論の解析法には, 変位成分を独立関数とする.解析法 (以後, 変位法ということにする) と応力関数を独立関数とする解 析法 (以後, 応力関数法ということにする) があるが, せん断変形 を含む板の曲げ理論には，変位法とたわみ及びせん断力を表す応 力関数を使用する解析法 (以後, 混合法ということにする) はある が, 応力関数法の報告は未だ行われていない，その理由は, 平面 応力理論においては, 応力関数を導入することによって, 独立関 数の数の少ない簡便な解析法の構筑が可能になるが, 板の曲げ理 論においては, 応力関数の導入が, 独立関数の隇少にはつながる ものの，簡便さにはつながらないという事情によるものと思われ る.

板の曲げ問題の解析に解析的な解のみを使用することを考える ならば, 応力関数法は変位法に較べて有利な立場にはない. しか し, 解析的な解法には積分可能条件を基礎式に加える必要のある 応力関数法と, 微分可能条件を加えなくても適応範囲の広い解が 得られる変位法との差を確認できるという学術的な価值がある.

本報告は, Hellinger-Reissner の原理のための沉関数を使用す る変分原理によって誘導された, せん断変形を考慮する板の曲げ 理論の基礎式を基にして, 登坂, 末岡 [7]によって誘導された新 しい応力関数を独立関数とする解析法 (以後, 応力関数法という) の定式化を行うとともに，Fourier 級数解を求め, 数值解析例の 解析結果を変位法による結果と比較することによって, 解析法の 妥当性を検討したものである.
応力関数法が構築されたことにより, 応力関数と変位を独立関 数とする Hellinger-Reissner の原理のための汎関数を使用する数 值解析法において応力関数の合理的な選定が可能になる.

\section{2. 新しい応力関数の導入}

3 次元理論においては, Maxwell の応力関数と Morera の 応力関数が知られているが, 本報告では, 登坂, 末岡によって導 かれた新しい応力関数を使用することにする. 新しい応力関数を $L, M, N$ とすると Maxwell の応力関数及び Morera の応力関数 との関係は式.(1)のように表わされる.

$$
\begin{array}{lll}
\chi_{1}=\frac{\partial L}{\partial x}, & \chi_{2}=\frac{\partial M}{\partial y}, & \chi_{3}=\frac{\partial N}{\partial z} \\
\psi_{1}=\frac{\partial N}{\partial y}+\frac{\partial M}{\partial z}, & \psi_{2}=\frac{\partial L}{\partial z}+\frac{\partial N}{\partial x}, & \psi_{3}=\frac{\partial M}{\partial x}+\frac{\partial L}{\partial y}
\end{array}
$$

ここに, $\chi_{1}, \chi_{2}, \chi_{3}$ は Maxwell の応力関数であり, $\psi_{1}, \psi_{2}, \psi_{3}$ は Morera の応力関数である.

Maxwell の応力関数及び Morera の応力関数を使用すると応力 は式 (2)のように表される.

$$
\sigma_{x}=\frac{1}{2}\left[\frac{\partial^{2} \chi_{3}}{\partial y^{2}}+\frac{\partial^{2} \chi_{2}}{\partial z^{2}}+\frac{\partial^{2} \psi_{1}}{\partial y \partial z}\right]
$$

\footnotetext{
* 長崎大学工学部構造工学科 教授. 工博

** 長崎大学工学部構造工学科 助手. 修士(工学)

*** 広島大学工学部第四類 助教授. 工博
}

Prof., Dept. of Structural Engineering, Faculty of Engineering, Nagasaki Univ., Dr. Eng.

Research Assoc., Dept. of Structural Engineering, Faculty of Engineering, Nagasaki Univ., M. Eng.

Assoc. Prof., Dept. of Structural Engineering, Faculty of Engineering, Hiroshima Univ., Dr. Eng. 


$$
\begin{aligned}
& \sigma_{y}=\frac{1}{2}\left[\frac{\partial^{2} \chi_{1}}{\partial z^{2}}+\frac{\partial^{2} \chi_{3}}{\partial x^{2}}+\frac{\partial^{2} \psi_{2}}{\partial z \partial x}\right] \\
& \sigma_{z}=\frac{1}{2}\left[\frac{\partial^{2} \chi_{2}}{\partial x^{2}}+\frac{\partial^{2} \chi_{1}}{\partial y^{2}}+\frac{\partial^{2} \psi_{3}}{\partial x \partial y}\right] \\
& \tau_{y z}=-\frac{1}{2}\left[\frac{\partial^{2} \chi_{1}}{\partial y \partial z}+\frac{1}{2} \frac{\partial}{\partial x}\left(-\frac{\partial \psi_{1}}{\partial x}+\frac{\partial \psi_{2}}{\partial y}+\frac{\partial \psi_{3}}{\partial z}\right)\right] \\
& \tau_{z x}=-\frac{1}{2}\left[\frac{\partial^{2} \chi_{2}}{\partial z \partial x}+\frac{1}{2} \frac{\partial}{\partial y}\left(\frac{\partial \psi_{1}}{\partial x}-\frac{\partial \psi_{2}}{\partial y}+\frac{\partial \psi_{3}}{\partial z}\right)\right] \\
& \tau_{x y}=-\frac{1}{2}\left[\frac{\partial^{2} \chi_{3}}{\partial x \partial y}+\frac{1}{2} \frac{\partial}{\partial z}\left(\frac{\partial \psi_{1}}{\partial x}+\frac{\partial \psi_{2}}{\partial y}-\frac{\partial \psi_{3}}{\partial z}\right)\right]
\end{aligned}
$$

新しい応力関数を使用すれば，Maxwell の応力関数で表示した 応力と Morera の応力関数で表示した応力の表示が等しくなり, 応力は式 (3)のように表される:

$$
\begin{aligned}
\sigma_{x} & =\frac{\partial^{2}}{\partial y \partial z}\left(\frac{\partial N}{\partial y}+\frac{\partial M}{\partial z}\right) \\
\sigma_{y} & =\frac{\partial^{2}}{\partial z \partial x}\left(\frac{\partial L}{\partial z}+\frac{\partial N}{\partial x}\right) \\
\sigma_{z} & =\frac{\partial^{2}}{\partial x \partial y}\left(\frac{\partial M}{\partial x}+\frac{\partial L}{\partial y}\right) \\
\tau_{y z} & =-\frac{\partial^{3} L}{\partial x \partial y \partial z} \\
\tau_{z x} & =-\frac{\partial^{3} M}{\partial x \partial y \partial z} \\
\tau_{x y} & =-\frac{\partial^{3} N}{\partial x \partial y \partial z}
\end{aligned}
$$

ここに, $\sigma_{x}, \sigma_{y}, \sigma_{z}$ 及び $\tau_{y z}, \tau_{z x}, \tau_{x y}$ は図 1 に示す垂直応力成 分, せん断応力成分である.

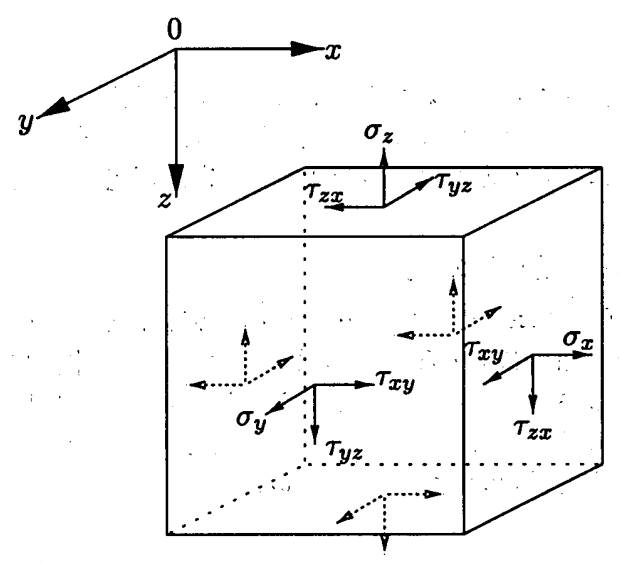

図 1. 底力成分

新しい応力関数による応力の表示式 (3) は Maxwell の応力関数 及び Morera の応力関数による応力の表示式 (2) に較べて簡明で ある.

板の上・下面における境界条件は式 (4)で与えられる力学的条 件とする.

$$
\begin{aligned}
& \left.\tau_{y z}\right|_{z= \pm h / 2}=0,\left.\quad \tau_{z x}\right|_{z= \pm h / 2}=0 \\
& \left.\sigma_{z}\right|_{z= \pm h / 2}= \pm \frac{p}{2}
\end{aligned}
$$

ここに, $\sigma_{z}$ は板の表面に垂直な応力, $\tau_{y z}, \tau_{z x}$ はせん断応力, $p$ は板の面に垂直な荷重であり， $h$ は板の厚さである.

既存のせん断変形を含む板の理論における応力と同じ表示を得 るために応力関数 $L, M, N$ を式 (5)のように仮定する.

$$
\begin{aligned}
& L=\left(\frac{3}{2 h}\right) z\left[1-\frac{1}{3}\left(\frac{z}{h / 2}\right)^{2}\right] L_{2}(x, y) \\
& M=\left(\frac{3}{2 h}\right) z\left[1-\frac{1}{3}\left(\frac{z}{h / 2}\right)^{2}\right] M_{2}(x, y) \\
& N=z N_{1}(x, y)+\frac{z^{2}}{2} N_{2}(x, y)
\end{aligned}
$$

式 (5) における応力関数 $N_{1}$ は平面応力理論における Airy の 応力関数に相当するものであるため, 板の曲げ問題の解析には不 要である. 従って，以後 $N_{1}$ の項は省略することにする.

式 (5) を式 (3) に代入すると, 式 (6) が得られる.

$$
\begin{aligned}
\sigma_{x} & =z\left[\frac{\partial^{2} N_{2}}{\partial y^{2}}-I^{-1} \frac{\partial M_{2}}{\partial y}\right] \\
\sigma_{y} & =z\left[\frac{\partial^{2} N_{2}}{\partial x^{2}}-I^{-1} \frac{\partial L_{2}}{\partial x}\right] \\
\sigma_{z} & =\left(\frac{3}{2 h}\right) z\left[1-\frac{1}{3}\left(\frac{z}{h / 2}\right)^{2}\right] p \\
\tau_{y z} & =-\left(\frac{3}{2 h}\right)\left[1-\left(\frac{z}{h / 2}\right)^{2}\right] \frac{\partial^{2} L_{2}}{\partial x \partial y} \\
\tau_{z x} & =-\left(\frac{3}{2 h}\right)\left[1-\left(\frac{z}{h / 2}\right)^{2}\right] \frac{\partial^{2} M_{2}}{\partial x \partial y} \\
\tau_{x y} & =-z \frac{\partial^{2} N_{2}}{\partial x \partial y}
\end{aligned}
$$

ここに, $I=h^{3} / 12$ 、である.

応力関数 $L_{2}, M_{2}$ と荷重 $p$ の間には式 (7) が成立する.

$$
\frac{\partial^{2}}{\partial x \partial y}\left(\frac{\partial M_{2}}{\partial x}+\frac{\partial L_{2}}{\partial y}\right)=p
$$

式 (6) における垂直応力 $\sigma_{z}$ は文献 [1] に示されている表示と異 なっている. 文献 [1]では, 板の上面・下面に作用する荷重を上 面のみに荷重 $p$ が作用するとしているのに对して，本報告では， 上・下面に $p / 2$ ずつ作用するとしていることによるもので本質的 な違いはない。

3. 応力関数 $L_{2}, M_{2}$

荷重 $p$ が, 式(8)のように関数 $\phi$ によって表されるものと仮定 する.

$$
p=\frac{\partial^{4} \phi}{\partial x^{2} \partial y^{2}}
$$

式 (8) を考慮すると微分方程式 (7) の解は次のようになる.

\section{1. 特殊解}

、特殊解は式 (9) のように求めることができる.

$$
L_{2 p}=\frac{1}{2} \frac{\partial \phi}{\partial x}, \quad M_{2 p}=\frac{1}{2} \frac{\partial \phi}{\partial y}
$$

\section{2. 一般解}

一般解は関数 $\Psi$ を用いて式 (10)のように表すことができる.

$$
L_{2 h}=\frac{\partial \Psi}{\partial x}, \quad M_{2 h}=-\frac{\partial \Psi}{\partial y}
$$

式 (9) 及び式 (10) より，応力関数 $L_{2}, M_{2}$ の解として式 (11) が 得られる。 


$$
\begin{aligned}
& L_{2}=\frac{1}{2} \frac{\partial \phi}{\partial x}+\frac{\partial \Psi}{\partial x}=\frac{\partial \Psi_{1}}{\partial x} \\
& M_{2}=\frac{1}{2} \frac{\partial \phi}{\partial y}-\frac{\partial \Psi}{\partial y}=-\frac{\partial \Psi_{2}}{\partial y} \\
& \text { ここに, } \Psi_{1}=\phi / 2+\Psi, \Psi_{2}=-\phi / 2+\Psi \text { である. }
\end{aligned}
$$

応力関数 $N_{2}, \Psi$ は後述する適合条件式から求めることになる.

\section{4. 合応力と応力関数の関係}

式 (11) を応力と応力関数の関係式 (6) に代入すると応力関数に よる応力の表示式 (12) が得られる.

$$
\begin{aligned}
\sigma_{x} & =z\left[\frac{\partial^{2} N_{2}}{\partial y^{2}}+I^{-1} \frac{\partial^{2} \Psi_{2}}{\partial y^{2}}\right] \\
\sigma_{y} & =z\left[\frac{\partial^{2} N_{2}}{\partial x^{2}}-I^{-1} \frac{\partial^{2} \Psi_{1}}{\partial x^{2}}\right] \\
\sigma_{z} & =\left(\frac{3}{2 h}\right) z\left[1-\frac{1}{3}\left(\frac{z}{h / 2}\right)^{2}\right] \frac{\partial^{4} \phi}{\partial x^{2} \partial y^{2}} \\
& =\left(\frac{3}{2 h}\right) z\left[1-\frac{1}{3}\left(\frac{z}{h / 2}\right)^{2}\right] p \\
\tau_{y z} & =-\left(\frac{3}{2 h}\right)\left[1-\left(\frac{z}{h / 2}\right)^{2}\right] \frac{\partial^{3} \Psi_{1}}{\partial x^{2} \partial y} \\
\tau_{z x} & =\left(\frac{3}{2 h}\right)\left[1-\left(\frac{z}{h / 2}\right)^{2}\right] \frac{\partial^{3} \Psi_{2}}{\partial x \partial y^{2}} \\
\tau_{x y} & =-z \frac{\partial^{2} N_{2}}{\partial x \partial y}
\end{aligned}
$$

式 (12) を使用して, 合応力 (以後, 一般化力ということにする) を求めると, 合応力と応力関数の関係式 (13) が得られる.

$$
\begin{aligned}
& M_{x}=I \frac{\partial^{2}}{\partial y^{2}}\left(N_{2}+I^{-1} \Psi_{2}\right) \\
& M_{y}=I \frac{\partial^{2}}{\partial x^{2}}\left(N_{2}-I^{-1} \dot{\Psi}_{1}\right) \\
& M_{x y}=-I \frac{\partial^{2} N_{2}}{\partial x \partial y} \\
& Q_{x}=\frac{\partial^{3} \Psi_{2}}{\partial x \partial y^{2}} \\
& Q_{y}=-\frac{\partial^{3} \Psi_{1}}{\partial x^{2} \partial y}
\end{aligned}
$$

ここに, $M_{x}, M_{y}$ は板の単位幅当りの曲げモーメント, $M_{x y}$ は 単位幅当りのねじりモーメント， $Q_{x}, Q_{y}$ は単位幅当りのせん 断力である. 図 2 に曲げモーメント $M_{x}, M_{y}$, ねじりモーメント $M_{x y}$, 世ん断力 $Q_{x}, Q_{y}$ の正の向きを示す.

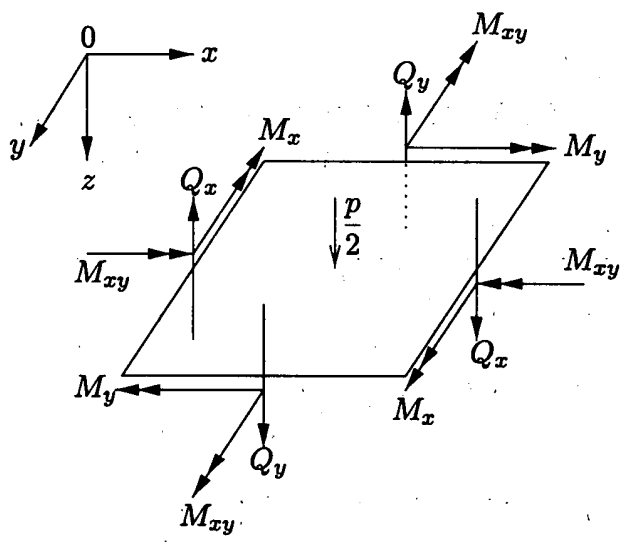

図 2. 合応力
式 (13) を式 (12) に代入すると, 応力と一般化力の関係式 (14) が得られる.

$$
\begin{aligned}
\sigma_{x} & =\frac{M_{x}}{I} z \\
\sigma_{y} & =\frac{M_{y}}{I} z \\
\sigma_{z} & =\left(\frac{3}{2 h}\right) z\left[1-\frac{1}{3}\left(\frac{z}{h / 2}\right)^{2}\right] \frac{\partial^{4} \dot{\phi}}{\partial x^{2} \partial y^{2}} \\
& =\left(\frac{3}{2 h}\right) z\left[1-\frac{1}{3}\left(\frac{z}{h / 2}\right)^{2}\right] p \\
\tau_{y z} & =\left(\frac{3}{2 h}\right)\left[1-\left(\frac{z}{h / 2}\right)^{2}\right] Q_{y} \\
\tau_{z x} & =\left(\frac{3}{2 h}\right)\left[1-\left(\frac{z}{h / 2}\right)^{2}\right]^{2} Q_{x} \\
\tau_{x y} & =\frac{M_{x y}}{I} z
\end{aligned}
$$

式 (14) は, 垂直応力 $\sigma_{z}$ の表示を除いて文献 [1]に示されてい るせん断変形を含む板の基礎式を誘導する際に使用されている応 力の仮定と同じである.

\section{5. 一般化変位と一般化ひずみの関係}

一般化力と一般化変位の関係は式(15)に示す HellingerReissner の原理のための沉関数を使用して求めることにする.

$$
\begin{aligned}
\Pi_{R}=\iiint_{V}[ & -B\left(\sigma_{x}, \cdots, \tau_{x y}\right) \\
& +\sigma_{x} \frac{\partial u}{\partial x}+\sigma_{y} \frac{\partial v}{\partial y}+\sigma_{z} \frac{\partial w}{\partial z} \\
& \quad+\tau_{y z}\left(\frac{\partial v}{\partial z}+\frac{\partial w}{\partial y}\right) \\
& +\tau_{z x}\left(\frac{\partial w}{\partial x}+\frac{\partial u}{\partial z}\right) \\
& \left.+\tau_{x y}\left(\frac{\partial u}{\partial y}+\frac{\partial v}{\partial x}\right)\right] d x d y d z \\
-\iint_{S_{u}} & {\left[p_{x}(u-\bar{u})+p_{y}(v-\bar{v})+p_{z}(w-\bar{w})\right] d z d s } \\
-\iint_{S_{\sigma}}\left[\overline{t_{x}} u+\overline{t_{y}} v+\overline{t_{z}} w\right] d z d s & -(\text { integration on } z= \pm h / 2)
\end{aligned}
$$

$$
\begin{aligned}
& B\left(\sigma_{x}, \cdots, \tau_{x y}\right)=\frac{1}{2 E}\{ {\left[\sigma_{x}-\nu\left(\sigma_{y}+\sigma_{z}\right)\right] \sigma_{x} } \\
&+\left[\sigma_{y}-\nu\left(\sigma_{z}+\sigma_{x}\right)\right] \sigma_{y} \\
&\left.+\left[\sigma_{z}-\nu\left(\sigma_{x}+\sigma_{y}\right)\right] \sigma_{z}\right\} \\
&+\frac{1}{2 G}\left\{\tau_{y z}^{2}+\tau_{z x}^{2}+\tau_{x y}^{2}\right\}
\end{aligned}
$$

ここに, $u, v, w$ は $x$ 軸, $y$ 軸及び $z$ 軸方向の正の向きを 正とする変位成分, $\overline{t_{x}}, \overline{t_{y}}, \overline{t_{z}}$ は板側面に働く単位面積当りの 表面力であり， $p_{x}, p_{y}, p_{z}$ は Lagrange の未定乗数である. $E$ はヤング係数， $G$ はせん断弾性係数，， $\boldsymbol{\nu}$ はポアソン比であり, $B\left(\sigma_{x}, \cdots, \tau_{x y}\right)$ はコンプリメンタリエネルギー関数である.

応力と一般化力の関係式 (14) を式 (15) に代入し, 厚さ方向に 積分すると, 汎関数として式 (17) が得られる. 


$$
\begin{aligned}
\Pi_{R}=\iint_{S}\{ & -\frac{1}{2 E I}\left[\left(M_{x}-\nu M_{y}\right) M_{x}+\left(M_{y}-\nu M_{x}\right) M_{y}\right. \\
& \left.+2(1+\nu) M_{x y}{ }^{2}-\frac{\nu h^{2}}{5} p\left(M_{x}+M_{y}\right)\right] \\
& -\frac{1}{2 G}\left[\frac{1}{\kappa h}\left(Q_{y}^{2}+Q_{x}^{2}\right)\right] \\
& +M_{x} \frac{\partial U_{1}}{\partial x}+M_{y} \frac{\partial V_{1}}{\partial y}-p W_{0} \\
& +Q_{y}\left(V_{1}+\frac{\partial W_{0}}{\partial y}\right) \\
& +Q_{x}\left(\frac{\partial W_{0}}{\partial x}+U_{1}\right) \\
& \left.+M_{x y}\left(\frac{\partial U_{1}}{\partial y}+\frac{\partial V_{1}}{\partial x}\right)\right\} d x d y \\
-\int_{S_{2}} & {\left[P_{x}\left(U_{1}-\overline{U_{1}}\right)\right.} \\
& +P_{y}\left(V_{1}-\overline{V_{1}}\right) \\
& \left.+P_{z}\left(W_{0}-\overline{W_{0}}\right)\right] d s \\
-\int_{S_{\sigma}} & {\left[\bar{M}_{x} U_{1}+\overline{M_{y}} V_{1}+\overline{Q_{n}} W_{0}\right] d s }
\end{aligned}
$$

ここに,

$$
\begin{aligned}
& \overline{M_{x}}=\int_{-h / 2}^{h / 2} \overline{t_{x}} z d z \\
& \overline{M_{y}}=\int_{-h / 2}^{h / 2} \overline{t_{y}} z d z \\
& \overline{Q_{n}}=\int_{-h / 2}^{h / 2} \overline{t_{z}} d z
\end{aligned}
$$

また, $P_{x}, P_{y}, P_{z}$ は Lagrange の未定乗数である.

式 (17) の停留条件式として，一般化力と一般化変位の関係式, 釣合条件式，幾何学的境界条件式及び力学的境界条件式が得られ るが, ここでは, 応力関数法の定式化に必要な一般化力と一般化 変位の関係式のみを式 (18)に示すことにする.

$$
\begin{aligned}
& M_{x}=D\left(\frac{\partial U_{1}}{\partial x}+\nu \frac{\partial V_{1}}{\partial y}\right)+\frac{\nu h^{2}}{10(1-\nu)} p \\
& M_{y}=D\left(\frac{\partial V_{1}}{\partial y}+\nu \frac{\partial U_{1}}{\partial x}\right)+\frac{\nu h^{2}}{10(1-\nu)} p \\
& M_{x y}=\frac{1-\nu}{2} D\left(\frac{\partial U_{1}}{\partial y}+\frac{\partial V_{1}}{\partial x}\right) \\
& Q_{x}=G \kappa h\left(U_{1}+\frac{\partial W_{0}}{\partial x}\right) \\
& Q_{y}=G \kappa h\left(V_{1}+\frac{\partial W_{0}}{\partial y}\right) .
\end{aligned}
$$

ここに, $D=E I /\left(1-\nu^{2}\right), \kappa=5 / 6$ であり, $U_{1}, V_{1}, W_{0}$ は式 (19) で定義される一般化変位である.

$$
\begin{aligned}
& U_{1}=\left(\frac{12}{h^{3}}\right) \int_{-h / 2}^{+h / 2} u z d z \\
& V_{1}=\left(\frac{12}{h^{3}}\right) \int_{-h / 2}^{+h / 2} v z d z \\
& W_{0}=\left(\frac{3}{2 h}\right) \int_{-h / 2}^{+h / 2} w\left[1-\left(\frac{z}{h / 2}\right)^{2}\right] d z
\end{aligned}
$$

一般化ひずみと一般化変位の関係を式 $(20)$ のように定義する.

$$
\begin{aligned}
& E_{x}=\frac{\partial U_{1}}{\partial x} \\
& E_{y}=\frac{\partial V_{1}}{\partial y} \\
& \Gamma_{x y}=\frac{\partial U_{1}}{\partial y}+\frac{\partial V_{1}}{\partial x} \\
& \Gamma_{y z}=V_{1}+\frac{\partial W_{0}}{\partial y} \\
& \Gamma_{z x}=U_{1}+\frac{\partial W_{0}}{\partial x}
\end{aligned}
$$

ここに, $E_{x}, E_{y}, \Gamma_{y z}, \Gamma_{z x}, \Gamma_{x y}$ は一般化ひずみである.

\section{6. 一般化ひずみと応力関数の関係}

式 (20)を考慮すると，式 (18)より一般化ひずみと一般化力の 関係式 (21) が得られる.

$$
\begin{aligned}
& E_{x}=\frac{1}{E I}\left[M_{x}-\nu M_{y}-\frac{\nu h^{2}}{10} p\right] \\
& E_{y}=\frac{1}{E I}\left[M_{y}-\nu M_{x}-\frac{\nu h^{2}}{10} p\right] \\
& \Gamma_{x y}=\frac{1}{G I} M_{x y} \\
& \Gamma_{y z}=\frac{Q_{y}}{G \kappa h} \\
& \Gamma_{z x}=\frac{Q_{x}}{G \kappa h}
\end{aligned}
$$

式 (21) に式 (13) を代入すると, 一般化ひずみと応力関数の関 係式 (22) が得られる.

$$
\begin{aligned}
E_{x} & =\frac{1}{E I}\left[\frac{\partial^{2}\left(I N_{2}+\Psi_{2}\right)}{\partial y^{2}}-\nu \frac{\partial^{2}\left(I N_{2}-\Psi_{1}\right)}{\partial x^{2}}-\frac{\partial^{4} \Phi}{\partial x^{2} \partial y^{2}}\right] \\
E_{y} & =\frac{1}{E I}\left[\frac{\partial^{2}\left(I N_{2}-\Psi_{1}\right)}{\partial x^{2}}-\nu \frac{\partial^{2}\left(I N_{2}+\Psi_{2}\right)}{\partial y^{2}}-\frac{\partial^{4} \Phi}{\partial x^{2} \partial y^{2}}\right] \\
\Gamma_{x y} & =-\frac{1}{G} \frac{\partial^{2} N_{2}}{\partial x \partial y} \\
\Gamma_{y z} & =-\frac{1}{G \kappa h} \frac{\partial^{3} \Psi_{1}}{\partial x^{2} \partial y} \\
\Gamma_{z x} & =\frac{1}{G \kappa h} \frac{\partial^{3} \Psi_{2}}{\partial x \partial y^{2}} \\
\Phi & =\frac{\nu h^{2}}{10} \phi
\end{aligned}
$$

\section{7. 適合条件式}

一般化ひずみと一般化変位の関係式 $(20)$ から一般化変位 $U_{1}$, $V_{1}, W_{0}$ を消去すると，適合条件式として式 (24) が得られる.

$$
\begin{aligned}
& R_{z}=\frac{\partial^{2} E_{x}}{\partial y^{2}}+\frac{\partial^{2} E_{y}}{\partial x^{2}}-\frac{\partial^{2} \Gamma_{x y}}{\partial x \partial y} \\
& U_{x}=-\frac{\partial E_{x}}{\partial y}+\frac{1}{2} \frac{\partial}{\partial x}\left[-\frac{\partial \Gamma_{y z}}{\partial x}+\frac{\partial \Gamma_{z x}}{\partial y}+\Gamma_{x y}\right] \\
& U_{y}=-\frac{\partial E_{y}}{\partial x}+\frac{1}{2} \frac{\partial}{\partial y}\left[\frac{\partial \Gamma_{y z}}{\partial x}-\frac{\partial \Gamma_{z x}}{\partial y}+\Gamma_{x y}\right]
\end{aligned}
$$

式 (24b) を $y$ で微分した式と，式 (24c) を $x$ で微分した式の和 及び差を求め式 $(24 \mathrm{a})$ を考慮すると, 適合条件式として式 $(25)$ が 得られる. 


$$
\begin{aligned}
& \frac{\partial^{2} E_{x}}{\partial y^{2}}+\frac{\partial^{2} E_{y}}{\partial x^{2}}-\frac{\partial^{2} \Gamma_{x y}}{\partial x \partial y}=0 \\
& \frac{\partial^{2} E_{x}}{\partial y^{2}}-\frac{\partial^{2} E_{y}}{\partial x^{2}}+\frac{\partial^{2}}{\partial x \partial y}\left[\frac{\partial \Gamma_{y z}}{\partial x}-\frac{\partial \Gamma_{z x}}{\partial y}\right]=0
\end{aligned}
$$

式 (22) を式 (25) に代入すると, 応力関数で表した適合条件式 (26) が得られる.

$$
\begin{array}{r}
I\left\{\frac{\partial^{4} N_{2}}{\partial x^{4}}+2 \frac{\partial^{4} N_{2}}{\partial x^{2} \partial y^{2}}+\frac{\partial^{4} N_{2}}{\partial y^{4}}\right\}+\left\{\frac{\partial^{4} \Psi}{\partial y^{4}}-\frac{\partial^{4} \Psi}{\partial x^{4}}\right\} \\
=\frac{1}{2}\left[\frac{\partial^{4} \phi}{\partial x^{4}}+\frac{\partial^{4} \phi}{\partial y^{4}}-2 \nu \frac{\partial^{4} \phi}{\partial x^{2} \partial y^{2}}\right. \\
\left.+\frac{\nu h^{2}}{5} \frac{\partial^{4}}{\partial x^{2} \partial y^{2}}\left(\frac{\partial^{2} \phi}{\partial x^{2}}+\frac{\partial^{2} \phi}{\partial y^{2}}\right)\right] \\
I\left\{-\frac{\partial^{4} N_{2}}{\partial x^{4}}+\frac{\partial^{4} N_{2}}{\partial y^{4}}\right\}+\left\{\frac{\partial^{4} \Psi}{\partial x^{4}}+2 \nu \frac{\partial^{4} \Psi}{\partial x^{2} \partial y^{2}}+\frac{\partial^{4} \Psi}{\partial y^{4}}\right\} \\
-\frac{E I}{G \kappa h} \frac{\partial^{4}}{\partial x^{2} \partial y^{2}}\left\{\frac{\partial^{2} \Psi}{\partial x^{2}}+\frac{\partial^{2} \Psi}{\partial y^{2}}\right\} \\
=\frac{1}{2}\left[-\frac{\partial^{4} \phi}{\partial x^{4}}+\frac{\partial^{4} \phi}{\partial y^{4}}+\frac{h^{2}}{5} \frac{\partial^{4}}{\partial x^{2} \partial y^{2}}\left(\frac{\partial^{2} \phi}{\partial x^{2}}-\frac{\partial^{2} \phi}{\partial y^{2}}\right)\right]
\end{array}
$$

式 (26) は, 一般化力と応力関数の関係式 (13) t HellingerReissner の原理のための汎関数である式 (17) に代入して得られ る Appendix A に示す汎関数を部分積分し, 応力関数 $N_{2}, \Psi$ に 関する停留条件式として求めることもできる.

\section{8. 変位の計算}

一般化変位と一般化ひずみの関係式 $(20)$ より, 変位は式 (27) のようになる。

$$
\begin{array}{r}
U_{1}=\frac{1}{E I}\left[\int \frac{\partial^{2}\left(I N_{2}+\Psi_{2}\right)}{\partial y^{2}} d x-\nu \frac{\partial\left(I N_{2}-\Psi_{1}\right)}{\partial x}\right. \\
\left.-\frac{\partial^{3} \Phi}{\partial x \partial y^{2}}+g_{1}(y)\right] \\
V_{1}=\frac{1}{E I}\left[\int \frac{\partial^{2}\left(I N_{2}-\Psi_{1}\right)}{\partial x^{2}} d y-\nu \frac{\partial\left(I N_{2}+\Psi_{2}\right)}{\partial y}\right. \\
\left.-\frac{\partial^{3} \Phi}{\partial x^{2} \partial y}+f_{1}(x)\right]
\end{array}
$$

式 (27) における関数 $f_{1}(x), g_{1}(y)$ は式 $(28)$ の解として求めら れる.

$$
\begin{array}{r}
\frac{\partial}{\partial y}\left[\int \frac{\partial^{2}\left(I N_{2}+\Psi_{2}\right)}{\partial y^{2}} d x-\nu \frac{\partial\left(I N_{2}-\Psi_{2}\right)}{\partial x}-\frac{\partial^{3} \Phi}{\partial x \partial y^{2}}\right] \\
+\frac{\partial}{\partial x}\left[\int \frac{\partial^{2}\left(I N_{2}-\Psi_{1}\right)}{\partial x^{2}} d y-\nu \frac{\partial\left(I N_{2}+\Psi_{2}\right)}{\partial y}-\frac{\partial^{3} \Phi}{\partial x^{2} \partial y}\right] \\
+\frac{d f_{1}(x)}{d x}+\frac{d g_{1}(y)}{d y} \\
=-2(1+\nu) I \frac{\partial^{2} N_{2}}{\partial x \partial y}
\end{array}
$$

変位 $W_{0}$ は式 $(29)$ によって求められる.

$$
\begin{aligned}
W_{0} & =-\left[\int V_{1} d y+\frac{1}{G \kappa h} \frac{\partial^{2} \Psi_{1}}{\partial x^{2}}+f_{2}(x)\right] \\
& =-\left[\int U_{1} d x-\frac{1}{G \kappa h} \frac{\partial^{2} \Psi_{2}}{\partial y^{2}}+g_{2}(y)\right]
\end{aligned}
$$

\section{9. 基礎微分方程式の Fourier 級数解}

\section{1. 特殊解}

特殊解として二重級数解を使用することにする。

$N_{2}$ を $N$ とおき, 応力関数を式 $(30)$ のように仮定する.

$$
\begin{aligned}
& N=\sum \sum A_{m n} \sin \lambda_{m} x \sin \lambda_{n} y \\
& \Psi=\sum \sum B_{m n} \sin \lambda_{m} x \sin \lambda_{n} y
\end{aligned}
$$

ここに, $A_{m n}, B_{m n}$ は未定係数であり, $\lambda_{m}=m \pi / a, \lambda_{n}=$ $n \pi / b$ である.

$$
\phi=\iiint \int p d x d x d y d y
$$

荷重 $p$ が式 (32) のように表されているものと仮定する.

$p=\sum \sum p_{m n} \sin \lambda_{m} x \sin \lambda_{n} y$

式 (30) 及び式 (31) を微分方程式 (26) に代入すれば未定係数 $A_{m n}, B_{m n}$ を求めることができる.

一般化力及び一般化変位は式 (33) のように求められる.

$$
\begin{aligned}
& M_{x}=\sum \sum\left\{\frac{\lambda_{m}^{2}+\nu \lambda_{n}^{2}}{\left(\lambda_{m}^{2}+\lambda_{n}^{2}\right)^{2}}+\frac{\nu h^{2}}{10} \frac{\lambda_{n}^{2}}{\lambda_{m}^{2}+\lambda_{n}^{2}}\right\} p_{m n} \\
& M_{y}=\sum \sum\left\{\frac{\nu \lambda_{m}^{2}+\lambda_{n}^{2}}{\left(\lambda_{m}^{2}+\lambda_{n}^{2}\right)^{2}}+\frac{\nu h^{2}}{10} \frac{\lambda_{m}^{2}}{\lambda_{m}^{2}+\lambda_{n}^{2}}\right\} p_{m n} \\
& M_{x y}=\sum \sum\left\{-\frac{(1-\nu) \lambda_{m} \lambda_{n}}{\left(\lambda_{m}^{2}+\lambda_{n}^{2}\right)^{2}}+\frac{\nu h^{2}}{10} \frac{\lambda_{m} \lambda_{n}}{\lambda_{m}^{2}+\lambda_{n}^{2}}\right\} p_{m n} \\
& \times \cos \lambda_{m} x \cos \lambda_{n} y \\
& Q_{x}=\sum \sum \frac{\lambda_{m}}{\lambda_{m}^{2}+\lambda_{n}^{2}} p_{m n} \cos \lambda_{m} x \sin \lambda_{n} y \\
& Q_{y}=\sum \sum \frac{\lambda_{n}}{\lambda_{m}^{2}+\lambda_{n}^{2}} p_{m n} \sin \lambda_{m}^{\prime} x \cos \lambda_{n} y \\
& U_{1}=\frac{1}{D} \sum \sum\left\{-\frac{\lambda_{m}}{\left(\lambda_{m}^{2}+\lambda_{n}^{2}\right)^{2}}+\frac{\nu h^{2}}{10(1-\nu)} \frac{\lambda_{m}}{\lambda_{m}^{2}+\lambda_{n}^{2}}\right\} p_{m n} \\
& V_{1}=\frac{1}{D} \sum \sum\left\{-\frac{\lambda_{n}}{\left(\lambda_{m}^{2}+\lambda_{n}^{2}\right)^{2}}+\frac{\nu h^{2}}{10(1-\nu)} \frac{\lambda_{n}}{\lambda_{m}^{2}+\lambda_{n}^{2}}\right\} p_{m n} \\
& \times \sin \lambda_{m} x \cos \lambda_{n} y \\
& W_{0}=\frac{1}{D} \sum \sum\left\{\frac{1}{\left(\lambda_{m}^{2}+\lambda_{n}^{2}\right)^{2}}+\frac{(2-\nu) h^{2}}{10(1-\nu)} \frac{1}{\lambda_{m}^{2}+\lambda_{n}^{2}}\right\} p_{m n} \\
& \times \sin \lambda_{m} x \sin \lambda_{n} y
\end{aligned}
$$

式 (33) は変位法の結果と完全に一致している.

\section{2. 一般解}

$\Psi_{1}=\Psi_{2}=\Psi$ である. $N_{2}=N$ とおき， $\Psi, N$ を式 (34)のよ うに仮定する.

$N=\sum N_{m} \sin \lambda_{m} x$
$\Psi=\sum \Psi_{m} \sin \lambda_{m} x$

式 (34) を微分方程式 (26) に代入すると, 式 (35) が得られる. 


$$
\begin{gathered}
I\left\{\lambda_{m}^{4} N_{m}-2 \lambda_{m}^{2} \frac{d^{2} N_{m}}{d y^{2}}+\frac{d^{4} N_{m}}{d y^{4}}\right\}+\left\{\frac{d^{4} \Psi_{m}}{d y^{4}}-\lambda_{m}^{4} \Psi_{m}\right\}=0 \\
I\left\{-\lambda_{m}^{4} N_{m}+\frac{d^{4} N_{m}}{d y^{4}}\right\}+\left\{\lambda_{m}^{4} \Psi_{m}-2 \nu \lambda_{m}^{2} \frac{d^{2} \Psi_{m}}{d y^{2}}+\frac{d^{4} \Psi_{m}}{d y^{4}}\right\} \\
+\frac{E I}{G \kappa h}\left(\lambda_{m}^{2}\right) \frac{d^{2}}{d y^{2}}\left\{-\lambda_{m}^{2} \Psi_{m}+\frac{d^{2} \Psi_{m}}{d y^{2}}\right\}=0 \\
\frac{E I}{G \kappa h}=\frac{(1+\nu) h^{2}}{5}
\end{gathered}
$$

式(35)、の解は式 (36)のように表すことができる.

$N_{m}=A_{m} e^{k y}, \quad \Psi_{m}=B_{m} e^{k y}$

式 (36) を微分方程式 (35) に代入し, 積分定数 $A_{m} \neq 0, B_{m} \neq$ 0 とすると特性方程式(37) が得られる:

$$
\left(k^{2}-\lambda_{m}^{2}\right)^{2} k^{2}\left\{-2+\frac{h^{2}}{5}\left(k^{2}-\lambda_{m}^{2}\right)\right\}=0
$$

特性値を式 (38)のように表す。

$$
\begin{array}{ll}
k_{1}=0 & k_{2}=0 \\
k_{3}=-\alpha_{m}, & k_{4}=+\alpha_{m} . \\
k_{5}=-\lambda_{m}, & k_{6}=+\lambda_{m} . \\
k_{7}=-\lambda_{m}, & k_{8}=+\lambda_{m}
\end{array}
$$

応力関数は式 (39)のようになる。

$$
\begin{aligned}
N=\Sigma[ & A_{1 m}+A_{2 m} y \\
& +A_{3 m} \cosh \alpha_{m} y+A_{4 m} \sinh \alpha_{m} y \\
& +A_{5 m} \cosh \lambda_{m} y+A_{6 m} \sinh \lambda_{m} y \\
& \left.+A_{7 m} \lambda_{m} y \cosh \lambda_{m} y+A_{8 m} \lambda_{m} y \sinh \lambda_{m} y\right] \sin \lambda_{m} x \\
\Psi=\Sigma\left[\begin{array}{c} 
\\
B_{1 m}+B_{2 m} y \\
\end{array}\right. & +B_{3 m} \cosh \alpha_{m} y+B_{4 m} \sinh \alpha_{m} y \\
& +B_{5 m} \cosh \lambda_{m} y+B_{6 m} \sinh \lambda_{m} y \\
& \left.+B_{7 m} \lambda_{m} y \cosh \lambda_{m} y+B_{8 m} \lambda_{m} y \sinh \lambda_{m} y\right] \sin \lambda_{m} x
\end{aligned}
$$

式 (39) を微分方程式 (35) に代入すると，積分定数間の関係と して式 (40) が得られる.

$$
\begin{array}{ll}
B_{1 m}=I A_{1 m}, & B_{2 m}=I A_{2 m} \\
B_{3 m}=-\frac{5 I}{\left(5+h^{2} \lambda_{m}^{2}\right)} A_{3 m}, & B_{4 m}=-\frac{5 I}{\left(5+h^{2} \lambda_{m}^{2}\right)} A_{4 m} \\
B_{5 m}=-\frac{2 I}{(1-\nu)} A_{8 m}, & B_{6 m}=-\frac{2 I}{(1-\nu)} A_{7 m} \\
B_{7 m}=0, & B_{8 m}=0
\end{array}
$$
る.

一般化力及び一般化変位の一般解は式 (41) のように求められ

$$
\begin{aligned}
& M_{x}=\sum \lambda_{m}^{2}\left\{\left(I A_{4 m}+B_{4 m}\right) \rho_{m}^{2} \sinh \alpha_{m} y\right. \\
& +\left(I A_{3 m}+B_{3 m}\right) \rho_{m}^{2} \cosh \alpha_{m} y \\
& +\left(I A_{6 m}+2 I A_{7 m}+B_{6 m}\right) \sinh \lambda_{m} y \\
& +\left(I A_{5 m}+2 I A_{8 m}+B_{5 m}\right) \cosh \lambda_{m} y \\
& \left.+I A_{8 m} \lambda_{m} y \sinh \lambda_{m} y+I A_{7 m} \lambda_{m} y \cosh \lambda_{m} y\right\} \sin \lambda_{m} x \\
& M_{y}=-\sum \lambda_{m}{ }^{2}\left\{\left(I A_{4 m}-B 4\right) \sinh \alpha_{m} y+\left(I A_{3 m} \ddot{-} B_{3 m}\right) \cosh \alpha_{m} y\right. \\
& +\left(I A_{6 m}-B_{6 m}\right) \sinh \lambda_{m} y+\left(I A_{5 m}-B_{5 m}\right) \cosh \lambda_{m} y \\
& \left.+I A_{8 m} \lambda_{m} y \sinh \lambda_{m} y+I A_{7 m} \lambda_{m} y \cosh \lambda_{m} y\right\} \sin \lambda_{m} x \\
& M_{x y}=-\sum \lambda_{m}^{2}\left\{I A_{3 m} \rho_{m} \sinh \alpha_{m} y+I A_{4 m} \rho_{m} \cosh \alpha_{m} y\right. \\
& +I\left(A_{5 m}+A_{8 m}\right) \sinh \lambda_{m} y+I\left(A_{6 m}+A_{7 m}\right) \cosh \lambda_{m} y \\
& \left.+I A_{7 m} \lambda_{m} y \sinh \lambda_{m} y+I A_{8 m} \lambda_{m} y \cosh \lambda_{m} y\right\} \cos \lambda_{m} x \\
& Q_{x}=\sum \lambda_{m}{ }^{3}\left\{B_{4 m} \rho_{m}{ }^{2} \sinh \alpha_{m} y+B_{3 m} \rho_{m}{ }^{2} \cosh \alpha_{m} y\right. \\
& \left.+B_{6 m} \sinh \lambda_{m} y+B_{5 m} \cosh \lambda_{m} y\right\} \cos \lambda_{m} x \\
& Q_{y}=\sum \lambda_{m}{ }^{3}\left\{B_{3 m} \rho_{m} \sinh \alpha_{m} y+B_{4 m} \rho_{m} \cosh \alpha_{m} y\right. \\
& \left.+B_{5 m} \sinh \lambda_{m} y+B_{6 m} \cosh \lambda_{m} y\right\} \sin \lambda_{m} x \\
& U_{1}=-\frac{1}{E I} \sum \lambda_{m}\left\{\left[I A_{4 m}\left(\rho_{m}{ }^{2}+\nu\right)+B_{4 m}\left(\rho_{m}^{2}-\nu\right)\right] \sinh \alpha_{m} y\right. \\
& +\left[I A_{3 m}\left(\rho_{m}^{2}+\nu\right)+B_{3 m}\left(\rho_{m}^{2}-\nu\right)\right] \cosh \alpha_{m} y \\
& +\left[I(1+\nu) A_{6 m}+2 I A_{7 m}+(1-\nu) B_{6 m}\right] \sinh \lambda_{m} y \\
& +\left[I(1+\nu) A_{5 m}+2 I A_{8 m}+(1-\nu) B_{5 m}\right] \cosh \lambda_{m} y \\
& 1+I(1+\nu) A_{8 m} \lambda_{m} y \sinh \lambda_{m} y \\
& \left.+I(1+\nu) A_{7 m} \lambda_{m} y \cosh \lambda_{m} y\right\} \cos \lambda_{m} x \\
& V_{1}=-\frac{1}{E I} \sum \frac{\lambda_{m}}{\rho_{m}}\left\{\left[I A_{3 m}\left(\nu \rho_{m}{ }^{2}+1\right)+B_{3 m}\left(\nu \rho_{m}{ }^{2}-1\right)\right] \sinh \alpha_{m} y\right. \\
& +\left[I A_{4 m}\left(\nu \rho_{m}{ }^{2}+1\right)+B_{4 m}\left(\nu \rho_{m}{ }^{2}-1\right)\right] \cosh \alpha_{m} y \\
& +\left[I(1+\nu) A_{5 m} \rho_{m}-I(1-\nu) A_{8 m} \rho_{m}\right. \\
& \left.-(1-\nu) B_{5 m} \rho_{m}\right] \sinh \lambda_{m} y \\
& +\left[I(1+\nu) A_{6 m} \rho_{m}-I(1-\nu) A_{7 m} \rho_{m}\right. \\
& \left.-(1-\nu) B_{6 m} \rho_{m}\right] \cosh \lambda_{m} y \\
& +I(1+\nu) A_{7 m} \rho_{m} \lambda_{m} y \sinh \lambda_{m} y \\
& \left.+I(1+\nu) A_{8 m} \rho_{m} \lambda_{m} y \cosh \lambda_{m} y\right\} \sin \lambda_{m} x \\
& W_{0}=\frac{1}{E I} \Sigma\left\{\left[I(1+\nu) A_{6 m}+2 I A_{7 m}+(1-\nu) B_{6 m}\right.\right. \\
& \left.+\frac{(1+\nu) h^{2}}{5} B_{6 m} \lambda_{m}^{2}\right] \sinh \lambda_{m} y \\
& +\left[I(1+\nu) A_{5 m}+2 I A_{8 m}+(1-\nu) B_{5 m}\right. \\
& \left.+\frac{(1+\nu) h^{2}}{5} B_{5 m} \lambda_{m}^{2}\right] \cosh \lambda_{m} y \\
& +I(1+\nu) A_{8 m} \lambda_{m} y \sinh \lambda_{m} y \\
& \left.+I(1+\nu) A_{7 m} \lambda_{m} y \cosh \lambda_{m} y\right\} \sin \lambda_{m} x
\end{aligned}
$$


10. 特性值 $k=0$ の解について

特性値 $k=0$ の解として式 (42) 及び式 (43) が得られる.

$$
\begin{aligned}
& M_{x}=0 \\
& M_{y}=0 \\
& M_{x y}=-\sum \lambda_{m} B_{2 m} \cos \lambda_{m} x \\
& Q_{x}=0 \\
& Q_{y}=\sum \lambda_{m}^{2} B_{2 m} \sin \lambda_{m} x
\end{aligned}
$$

$$
\begin{aligned}
& U_{1}=g_{3}(y) \\
& V_{1}=f_{3}(x)
\end{aligned}
$$

一般化ひずみと一般化力の関係式 (21) を考慮し，式 (43)を一 般化ひずみと一般化変位の関係式 (20) に代入すると式 (44) が得 られる。

$$
\frac{d g_{3}(y)}{d y}+\frac{d f_{3}(x)}{d x}=-\frac{1}{G I} \lambda_{m} B_{2 m} \cos \lambda_{m} x
$$

式(44)より式(45)が得られる.

$B_{1 m}=0, \quad B_{2 m}=0, \quad \frac{d f_{3}(x)}{d x}+\frac{d g_{3}(y)}{d y}=0$

式 (45)の解は式 (46)のように表される.

$$
\begin{aligned}
& f_{3}(x)=b+c x \\
& g_{3}(y)=a-c y
\end{aligned}
$$

式 (46) における関数 $f_{3}(x), g_{3}(y)$ は式 $(27)$ における特性值 $k \neq 0$ の解より得られる変位に含まれている関数 $f_{1}(x), g_{1}(y) に$ 含ませることができる. 従って, 特性值 $k=0$ の解は不要にな る.

正解であるから，一般解についても皘分定数の変換を行なえ ば, 二重 Fourier 級数解の場合と同じょうに，変位及び応力につ いて変位法と同じ表示が得られるはずである。

\section{1. 数值解析例}

解析法の妥当性を検討するために，図 3 に示すような等分布荷 重を受ける相対する 2 辺が単純支持, 他の 1 辺が固定支持され, 残りの 1 辺が自由なる境界条件を有する長方形板の解析を行い, 変位法による解析結果との比較を行う．特殊解として使用する二 重 Fourier 級数解については変位法による解と応力関数法による 解が同じになることを確認している.

$x=0, a$ における境界条件は， $W_{0}=0, V_{1}=0, M_{x}=0$ であ るが, $y=0, b$ おける境界条件は式 (47) に示すとおりとする. 特 殊解として使用する二重 Fourier 級数解については $x$ 軸及び $y$ 軸 方向の展開項数を $m=n=15$, 一般解については $x$ 軸方向の展 開項数を $m=15$ として計算した結果を表 1 に示す.

\begin{tabular}{|c|c|c|c|c|c|c|}
\hline$x=0$ & $y=0$ & $y=b / 4$ & $y=b / 2$ & $y=\frac{3}{4} b$ & $y=b$ & 単位 \\
\hline$U_{1 D}$ & 0 & $-3,4602 e-03$ & $-6.9553 \theta-03$ & $-8.7922 \mathrm{e}-03$ & $-1.0327 \mathrm{e}-02$ & $p a^{4} / D$ \\
\hline$U_{1 s}$ & 0 & $-3.4602 \theta-03$ & $-6.95530-03$ & $-8.7922 \theta-03$ & $-1.0327 \mathrm{e}-02$ & $p a^{4} / D$ \\
\hline$M_{x y D}$ & $-3.7108 \theta-03$ & $-3.4521 \theta-02$ & $-2.0454 \theta-02$ & $-1.0822 a-02$ & 0 & $p a^{2}$ \\
\hline$M_{x y s}$ & $-3.7108 \mathrm{e}-03$ & $-3.4521 \mathrm{e}-02$ & $-2.0454 \theta-02$ & $-1.0822 \theta-02$ & 0 & $p a^{2}$ \\
\hline$Q_{x D}$ & 0 & $2.33810-01$ & $4.0132 \theta-01$ & $4.3283 e-01$ & $3.0541 \mathrm{e}+00$ & $p a$ \\
\hline$Q_{x S}$ & 0 & $2.3381 \mathrm{e}-01$ & $4.0132 \mathrm{e}-01$ & $4.3283 \mathrm{e}-01$ & $3.05410+00$ & $p a$ \\
\hline$x=\frac{a}{2}$ & $y=0$ & $y=b / 4$ & $y=b / 2$ & $y=\frac{3}{4} b$ & $y=b$ & 彗位 \\
\hline$V_{1 D}$ & 0 & $-3.6106 e-03$ & $-2.1676 \theta-03$ & $-1.1532 \theta-03$ & $-2.1883 \theta-03$ & $p a^{4} / D$ \\
\hline$V_{1 S}$ & 0 & $-3.6106 e-03$ & $-2.1676 \theta-03$ & $-1.1532 \theta-03$ & $-2.1883 \theta-03$ & $p a^{4} / D$ \\
\hline$W_{0 D}$ & 0 & $4.1956 \theta^{-03}$ & $8.6265 \theta-03$ & $1.0970 \theta-02$ & $1.3071 \mathrm{e}-02$ & $p a^{4} / D$ \\
\hline$W_{0 S}$ & 0 & 4.1956e-03 & $8.6265 \mathrm{e}-03$ & $1.09700-02$ & $1.3071 \mathrm{e}-02$ & $p a^{4} / D$ \\
\hline$M_{x D}$ & $-3.0702 a-02$ & $4.0232 e-02$ & $8.56400-02$ & $1.0554 \mathrm{e}-01$ & $1.1627 \mathrm{e}-01$ & $p a^{2}$ \\
\hline$M_{x S}$ & $-3.0702 e-02$ & $4.0232 \mathrm{e}-02$ & $8.5640 \mathrm{e}-02$ & $1.0554 \mathrm{e}-01$ & $1.1627 \mathrm{e}-01$ & $p a^{2}$ \\
\hline$M_{y_{D}}$ & $-1.2281 \mathrm{e}-01$ & $1.68700-02$ & $3.6670 \theta-02$ & $2.95510-02$ & 0 & $p a^{2}$ \\
\hline$M_{y_{S}}$ & $-1.2281 \mathrm{e}-01$ & $1.6870 \mathrm{e}-02$ & $3.66700-02$ & $2.9551 \mathrm{e}-02$ & 0 & $p a^{2}$ \\
\hline & $7.07100-01$ & $2.4154 e-01$ & $6.6844 \theta-02$ & $-5.7719 e-03$ & 0 & \\
\hline & $7.07100-01$ & $2.4154 \mathrm{e}-01$ & $6.6844 \mathrm{e}-02$ & $-5.7719 e-03$ & 0 & \\
\hline
\end{tabular}

$$
\begin{aligned}
& \left.U_{1}\right|_{y=0}=0,\left.\quad V_{1}\right|_{y=0}=0,\left.\quad W_{0}\right|_{y=0}=0 \\
& \left.M_{y}\right|_{y=b}=0,\left.\quad M_{x y}\right|_{y=b}=0,\left.\quad Q_{y}\right|_{y=b}=0
\end{aligned}
$$

上段に subscript $D$ を付けて変位法による結果を示し，下段に subscript $S$ を付けて応力法による結果を示してある。表1より 応力関数法による結果は変位法による結果と完全に一致している ことが認められる。

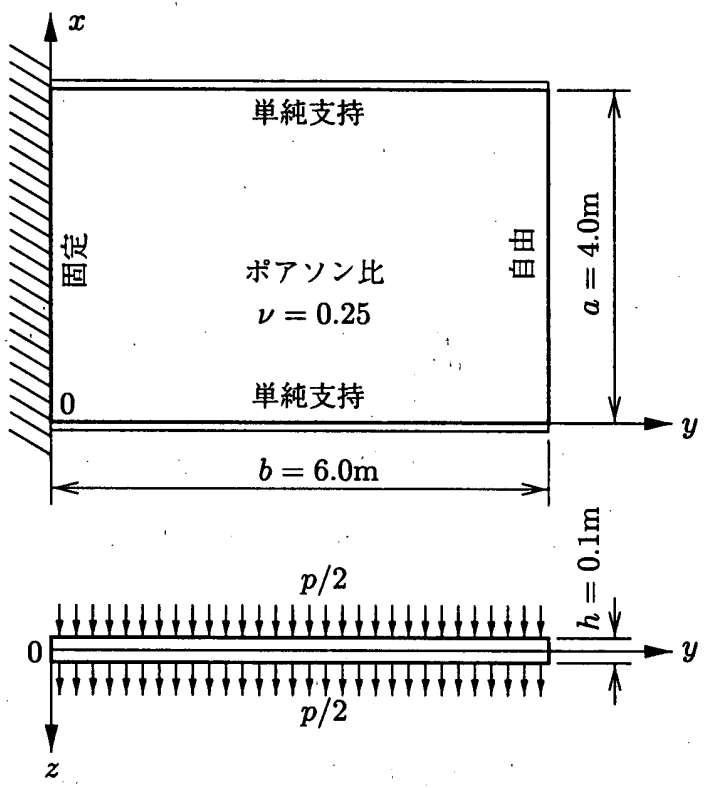

図 3. 解析モデル

表 1. 解析結果

12. 結び

Hellinger-Reissner の原理のための沉関数を使用して誘導され たせん断変形を含む板の曲げ理論の基礎式を基に，新しい応力関 数を独立関数とする解析法を構築した. 解析法の妥当性を検討す るために数值解析を行い，変位法による解析結果と比較してみた 結果次のことがわかった.

1. 新しい 3 個の応力関数 $L, M, N$ を使用したことにより, 従 来のように Maxwell の応力関数 3 個と Morera の応力関数 3 個の合計 6 個の応力関数を使用した場合に比べて, 応力関 数による応力の表示が簡明になり, Hellinger-Reissner の 原理から直接適合条件を求めることもできる明快な解析法 の構築が可能になった。

2. 一般解には，変位法にない特性值零の不要な解が含まれて いる，応力関数法では，応力及び変位を求めることによっ 
て解そのものを吟味することが必要になる.

3. 新しい応力関数を使用して求めた二重 Fourier 級数解によ る一般化変位及び一般化力は，変位法による結果と式のう えで完全に一致した。

4. 等分布荷重を受ける相対する 2 辺単純支持，他の. 1 辺固 定, 残る 1 辺自由なる境界を有する板の解析結果が, 変位 法による結果と一致したことから，新しい応力関数を使用 する解析法は妥当な解析法であると判断することができ る。

5. 新しい応力関数による応力の表示式は, 変位と応力関数を 独立関数とする Hellinger-Reissner の原理に基礎をおく有 限要素法にも使用することができる.

\section{参考文献}

[1] KYUICHIRO WASHIZU: VARIATIONAL METHODS IN ELASTICITY AND PLASTICITY, PERGAMON PRESS, PP. 11-13, PP.262-264, 1968.

[2] S.TIMOSHENKO; S.WOINOWSKY-KRIEGER: THEORY OF PLATES AND SHELLS, SECOND EDITION, MCGRAW-HILL, PP.165-171.

[3] 坪井義勝: 建築学体系 9-I 建築弾塑性学, 彰国社, pp. 110-112, 1969 .

[4] 坪井義勝: “連続体力学序説; 産業図書, pp. 75-86, 1977.

[5] 小松定夫: 構造解析学 III 一弾性連続体の解析 一, 丸善, pp. 202$210,1986$.

[6] 駕津久一郎: 弾性学の変分原理概論, コンピューターに上る構造工 学講座 II-3-A，日本銅構造協会編，.pp. 173-175, 1972.

[7] 登坂宣好, 末岡禎佑: 弾性体の応力関数, 日本建筑学会 1997 年度大 会 (関東) 学術講演梗概集, B-1, 構造 1， pp. 505-506, 1997.

[8] V.A. SALERNO, M.A. GOLDBERG: Effect of Shear Deformations on the Bending of Rectangular Plate, Transactions of the ASME, pp. 54-58, MARCH, 1960.

\section{Appendix A}

$$
\begin{aligned}
& \Pi_{R}=\iint_{S}\left\{-\frac{1}{2 E I}\left[\left(\frac{\partial^{2}\left(I N_{2}+\Psi_{2}\right)}{\partial y^{2}}-\nu \frac{\partial^{2}\left(I N_{2}-\Psi_{1}\right)}{\partial x^{2}}\right)\right.\right. \\
& \times \frac{\partial^{2}\left(I N_{2}+\Psi_{2}\right)}{\partial y^{2}} \\
& +\left(\frac{\partial^{2}\left(I N_{2}-\Psi_{1}\right)}{\partial x^{2}}-\nu \frac{\partial^{2}\left(I N_{2}+\Psi_{2}\right)}{\partial y^{2}}\right) \\
& \times \frac{\partial^{2}\left(I N_{2}-\Psi_{1}\right)}{\partial x^{2}} \\
& +2(1+\nu)\left(I \frac{\partial^{2} N_{2}}{\partial x \partial y}\right)^{2} \\
& \left.-\frac{\nu h^{2}}{5} p\left(\frac{\partial^{2}\left(I N_{2}+\Psi_{2}\right)}{\partial y^{2}}+\frac{\partial^{2}\left(I N_{2}-\Psi_{1}\right)}{\partial x^{2}}\right)\right] \\
& -\frac{1}{2 G} \frac{1}{\kappa h}\left[\left(\frac{\partial^{3} \Psi_{2}}{\partial x \partial y^{2}}\right)^{2}+\left(\frac{\partial^{3} \Psi_{1}}{\partial x^{2} \partial y}\right)^{2}\right] \\
& +\frac{\partial^{2}\left(I N_{2}+\Psi_{2}\right)}{\partial y^{2}} \frac{\partial U_{1}}{\partial x} \\
& +\frac{\partial^{2}\left(I N_{2}-\Psi_{1}\right)}{\partial x^{2}} \frac{\partial V_{1}}{\partial y} \\
& -\frac{\partial^{3} \Psi_{1}}{\partial x^{2} \partial y}\left(V_{1}+\frac{\partial W_{0}}{\partial y}\right) \\
& +\frac{\partial^{3} \Psi_{2}}{\partial x \partial y^{2}}\left(\frac{\partial W_{0}}{\partial x}+U_{1}\right) \\
& \left.-I \frac{\partial^{2} N_{2}}{\partial x \partial y}\left(\frac{\partial U_{1}}{\partial y}+\frac{\partial V_{1}}{\partial x}\right)-p W_{0}\right\} d x d y \\
& -\int_{S_{2}}\left[P_{x}\left(U_{1}-\overline{U_{1}}\right)\right. \\
& +P_{y}\left(V_{1}-\overline{V_{1}}\right) \\
& \left.+P_{z}\left(W_{0}-\overline{W_{0}}\right)\right] d s \\
& -\int_{S_{\sigma}}\left[\overline{M_{x}} U_{1}+\overline{M_{y}} V_{1}+\overline{Q_{n}} W_{0}\right] d s
\end{aligned}
$$

